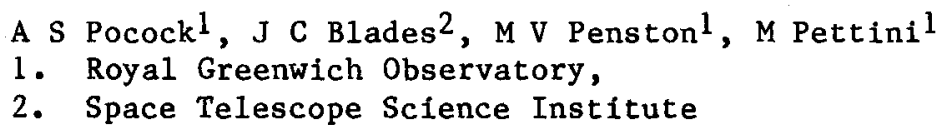

We have presented here some results of our search for quasars near large galaxies. Papers on these results are to be published shortly in Monthly Notices of the Royal Astronomical Society (Mon. Not. R. astr. Soc.)

\title{
1. OBJECTIVE
}

The aim of our programme is to use the absorption spectra of QSOs and related objects (BL Lacs, Seyfert galaxies) as probes of the interstellar media of intervening galaxies. For such a study it is necessary to find new suitably bright QSOs and related objects lying close on the sky to large galaxies because very few are known at present.

Since 1981 we have been carrying out a large scale search programme using UK Schmidt Telescope objective prism plates taken specifically for this purpose. Because we are looking for objects bright enough for high dispersion spectroscopy ( $>17.5 \mathrm{mag}(B)$ ), short exposure prism plates have been taken for us to a limiting magnitude of 17.5: on sky 1imited exposures, such bright QSOs are saturated and harder to identify. We have searched several fields and have taken follow-up slit spectra of candidates in the fields of 6 galaxies using the 74 inch reflector at the South Africal Astronomical Observatory (SAAO). A test of the hypothes is that QSO absorption systems arise in galaxy haloes is best made by looking at the absorption spectrum of background QSOs seen through the outer parts of nearby galaxies.

Recent studies show that nearby spirals can have extents of up to $100-200 \mathrm{kpc}$ in $\mathrm{HI}$.

\section{SEARCH PROCEDURE}

The spectra of QSOs typically show ultraviolet excess and/or emission lines. The search work is done by inspection of the objective 
prism plates by eye to look for those objects with ultraviolet excesses and/or emission lines. We have also searched for bright compact blue galaxies selected from the length of the spectra since this gives the required combined measure of blueness and compactness. These bright compact galaxies may also lie behind the foreground galaxies and if they are bright enough they may also be used to probe the interstellar media of foreground galaxies in just the same way as QSOs.

Once an object is selected from the objective prism plate, it is checked against the appropriate direct plate copy from the ESO B/SRC J sky survey to ensure that the candidate is a single object and its long spectrum not attributable to overlapping objects.

The problems encountered during the searching, are worse than in other QSO searches since we are looking for brighter QSOs, and are due to contamination by other types of objects besides QSOs which can also show ultraviolet excess or emission lines.

The types of objects which come into the above catagories are white dwarfs, O, B, A stars, planetary nebulae, cataclysmic variables and $M$ stars (which show apparent emission lines caused by gaps between absorption bands).

\section{RESULTS}

Follow up low resolution slit spectroscopy of QSO candidates was done on the 74 inch reflector at SAAO using the Reticon Photon Counting System (RPCS) and unit spectrograph with dispersion of $210 \AA / \mathrm{mm}$ to cover the spectral range $\sim 3200-7000 \AA$ with a slit of $1.8 \times 6$ arcseconds orientated $\mathrm{E} / \mathrm{W}$. The data has been reduced on the RGO Starlink VAX 11/780 using the SPICA reduction package. Interesting objects identified are:-

11 QSOs magnitudes 16-17(B)

2 Seyfert 1 galaxies, 1 Seyfert 2 galaxy

Several normal galaxies bright enough to use as probes

$M$ stars (2 of which are thought to be Mira variables)

Planetary nebula

1 cataclysmic variable (shows strong ultraviolet excess on prism plate) see an upcoming paper now in press with Mon. Not. R. astr. Soc. Echevarria, Pocock, Penston and Blades to be published Nov/Dec 1983.

CaII is the strongest interstellar line in the optical that we expect to see in absorption from an external galaxy accessible from ground telescopes. Other interstellar lines that are strong in ultraviolet and optical will eventually be observed using Space Telescope.

We shall look at the QSOs and Seyfert/bright galaxies at high resolution at the Anglo Australian Telescope (AAT) probing the interstellar medium of the foreground galaxies at different distances from the disk. 


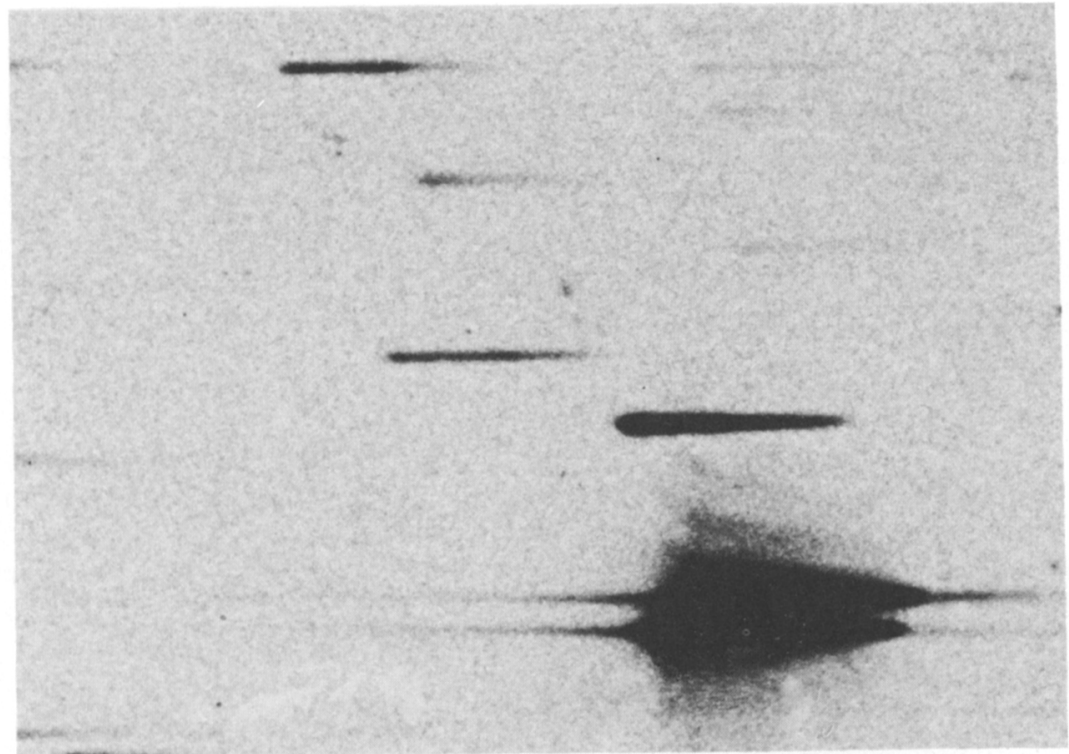

Plate 1

Objective prism spectrum of confirmed QSO 0050-254 (centre)

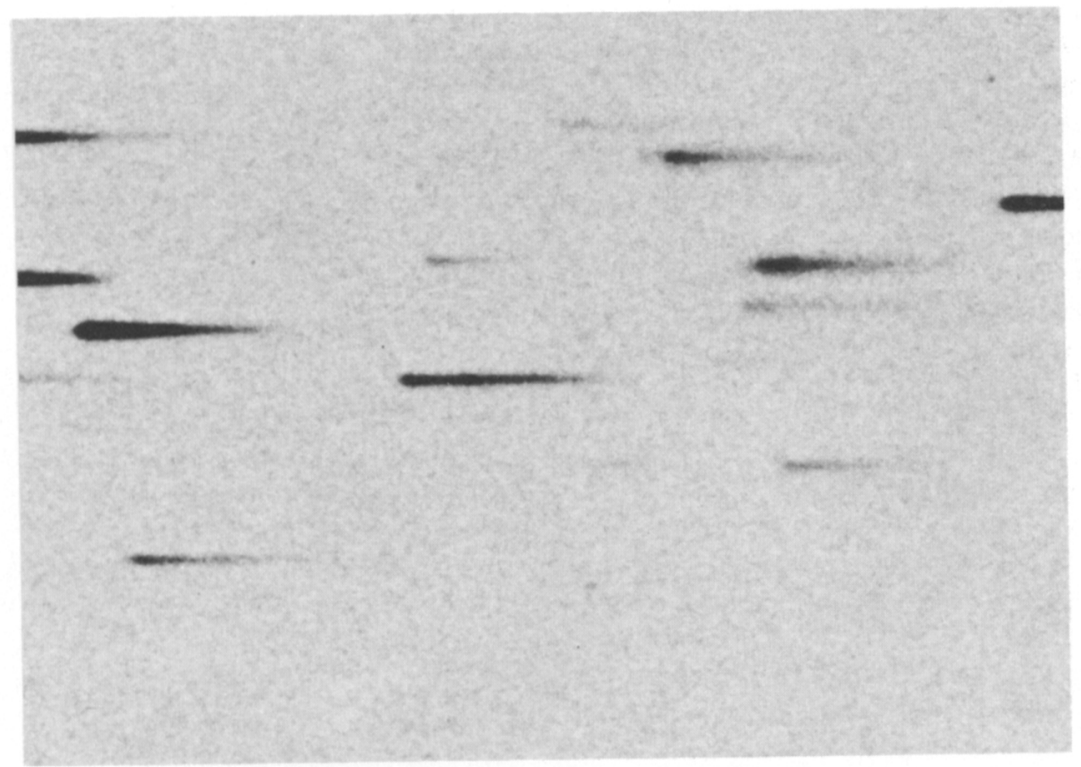

Plate 2

Objective prism spectrum of confirmed QSO 0041-261 (centre) 


\section{CONCLUSION}

The results confirm our ability to select QSO candidates. The point like candidates turned out to be mainly QSOs $(\sim 25 \%)$, white dwarfs, O, B, A stars, M stars, planetary nebula, cataclysmic variable.

It is expected that some of the candidates would be these other objects (ie stars) because of the higher contamination by such objects at the magnitude to which we are working $(\sim 17.5)$.

\section{FINAL REMARK}

Going through Schmidt and Green's Palomar Bright Quasar Survey: we have found that several of these QSOs are close on the sky (with less than 5-10 galaxy diameters) to foreground galaxies, some with known redshifts. For some of these nearby galaxies, we have measured the galaxy redshifts and we shall also use these QSOs to probe the interstellar media of the intervening galaxy in the same way.

The first paper will be submitted to Mon. Not. R. astr. Soc. in the coming months.

\section{DISCUSSION}

KUNTH: Since you wish to avoid getting high-z QSOs when aiming to further detect absorptions from intexvening galaxies, why do you use objective prism techniques and not other ones such as two colour techniques so as to get lower-z candidates?

POCOCK: We did not think that we would select mainly high-z QSOs by our method. We have, however, found some low-z QSOs and seyfert galaxies using the objective prism method. It would be ideal to obtain some two colour plates, and combine the two methods. 